\title{
Non-mydriatic fundus photography: a practical review for the neurologist
}

\author{
Devin D Mackay, ${ }^{1}$ Beau B Bruce ${ }^{2,3}$
}

\begin{abstract}
${ }^{1}$ Departments of Neurology, Ophthalmology, and

Neurosurgery, Indiana University School of Medicine, Indianapolis, Indiana, USA

${ }^{2}$ Departments of Ophthalmology and Neurology, Emory University School of Medicine, Atlanta, Georgia, USA

${ }^{3}$ Department of Epidemiology, Rollins School of Public Health and Laney Graduate School, Emory University, Atlanta, Georgia, USA
\end{abstract}

\section{Correspondence to}

Dr Beau B Bruce, Departments of Ophthalmology and Neurology, Neuro-

Ophthalmology Unit, Emory Eye Center, The Emory Clinic, Emory University School of Medicine, 1365-B Clifton Road NE, Atlanta, GA 30322, USA; bbbruce@emory.edu

Accepted 14 June 2016 Published Online First 12 July 2016

CrossMark

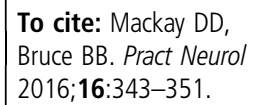

\begin{abstract}
Declining proficiency in direct ophthalmoscopy by non-ophthalmologists has spurred a search for alternative methods of ocular fundus examination. Recent technological advances have improved the ease of use and quality of nonmydriatic fundus photography, increasing its suitability for clinical care. As the availability of this technology continues to improve, neurologists will need to be familiar with its advantages, limitations and potential applications in the clinical care of patients with neurological conditions.
\end{abstract}

\section{INTRODUCTION}

Examination of the ocular fundus frequently yields important information that influences the clinical care of patients with neurological disease. However, difficulty performing ophthalmoscopy has contributed to the demise of ophthalmoscopy by non-ophthalmologists. ${ }^{1}$ The technical barriers of using a direct ophthalmoscope have led to renewed interest in alternative methods of viewing the optic disc, retina and retinal vessels. Technical advances coupled with the continued relevance of the funduscopic examination have driven interest in non-mydriatic fundus photography. Although it has been used since the 1970s, the technology has only recently advanced to the point of quickly obtaining high-quality fundus photographs, with minimal operator training and to rival the quality of mydriatic fundus cameras. Furthermore, research over the past decade has clarified its potential role in clinical care. As neurologists are among the most prominent non-ophthalmologists to gather information from the ocular fundus examination for use in clinical care, it is important that they are familiar with non-mydriatic fundus photography, its practical use in clinical settings, and conditions that may be more easily diagnosed or managed with its use.

\section{HOW DOES NON-MYDRIATIC FUNDUS PHOTOGRAPHY WORK?}

A cooperative patient sits in front of the fundus camera in a room with ambient lighting minimised (figure 1) or turned off. However, if the ambient lighting cannot be adjusted, the clinician may place an opaque cover supported by a frame over the front of the camera and over the patient's head and shoulders. The patient looks forward into the camera at a fixation light, and infrared fundus videography is used to focus on the region of interest. Many non-mydriatic cameras have software that automatically detects the posterior pole of the eye and takes a photograph when the back of the eye is in focus. Light in the infrared spectrum does not stimulate pupillary contraction. However, an infrared light source alone cannot give useful photographic images, and a flash is still needed. Sensitive image sensors in digital non-mydriatic fundus cameras allow the use of low flash settings. This helps to limit the amount of persistent pupillary constriction following the first image obtained with a flash, although the best quality pictures are obtained after an interphotographic interval of 30-60 s. ${ }^{2}$ Advances in digital photography technology have paved the way for additional features that make non-mydriatic fundus cameras easier to use, including autofocusing and autoalignment mechanisms, user interface software with task automation and the ability to print images, export them to a database or electronic medical record, or share them electronically using a network or internet connection.

\section{WHAT EVIDENCE SUPPORTS THE USE OF NON-MYDRIATIC FUNDUS PHOTOGRAPHY IN NON-OPHTHALMOLOGY SETTINGS?}

A growing body of research has evaluated the impact of non-mydriatic fundus 


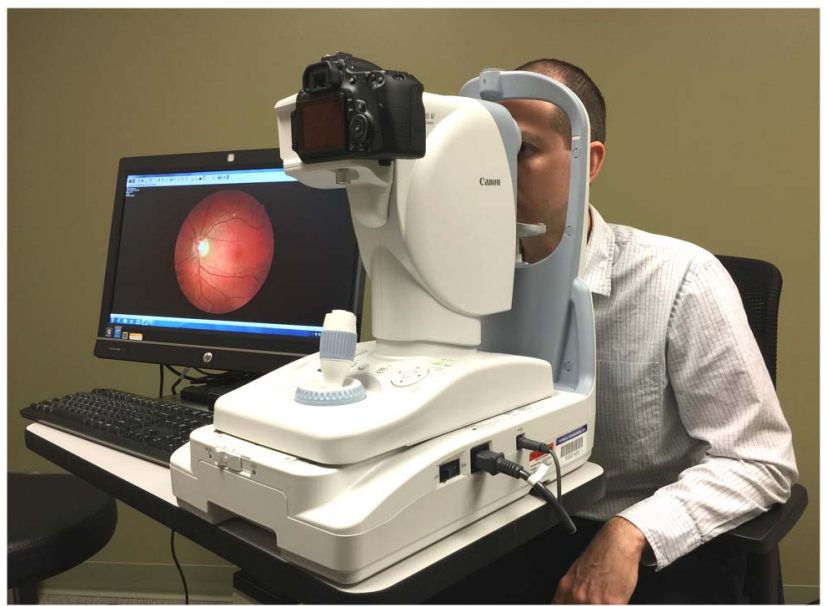

Figure 1 Typical configuration of non-mydriatic fundus camera. The patient is seated in a chair opposite the fundus camera. A computer is attached to the device, and runs software that automates portions of the image acquisition process and allows for digital image review, storage and distribution.

photography in the emergency department. A cohort of 350 adult patients was enrolled in the Fundus Photography versus Ophthalmoscopy Trial Outcomes in the Emergency Department (FOTO-ED) study. Inclusion criteria included a chief complaint of headache, acute focal neurological deficit, acute vision change or a diastolic blood pressure of at least $120 \mathrm{~mm} \mathrm{Hg}$. Forty-four of the 350 patients (13\%) had a relevant ocular finding on fundus photography (optic disc oedema, intraocular haemorrhage, severe hypertensive retinopathy, arterial vascular occlusion or optic disc pallor). Eleven of the 44 findings were known before the patients presented to the emergency department; of the remaining 33 findings, 6 were found during ophthalmology consultation and the other 27 were identified only by fundus photography. Only 5 of the 33 patients with relevant ocular findings that were not known before presentation underwent a funduscopic examination by an emergency physician, all of which were documented as normal. ${ }^{3}$

Phase I of the FOTO-ED study focused on the feasibility of non-mydriatic fundus photography in the emergency department and found that $83 \%$ of the 350 patients had a least one eye with a high-quality photograph. Nurse practitioners were given brief training and took the photographs used in the study, with a median image acquisition time of 1.9 min per session. Ease and speed of image acquisition were rated highly by the nurse practitioners and patients. ${ }^{4}$

Phase II of the FOTO-ED study focused on diagnostic accuracy and use of non-mydriatic fundus photography in the emergency department. ${ }^{5}$ In this phase, emergency physicians were given access to the photographs taken during the visit, and could use them to help influence patient care. Three hundred and fiftyfour patients were enrolled and $35(10 \%)$ were found to have relevant findings on fundus photographs. Emergency department physicians reviewed the photographs of 238 patients (68\%), identified 16 of the 35 relevant findings (46\%) using the photographs and reported them to be helpful for 125 patients $(35 \%)$.

In a separate study of patients with headache presenting to an academic emergency department, 8.5\% (42/497) had abnormal ocular fundus findings detected via non-mydriatic fundus photography. Fourteen of the 41 patients (41\%) with abnormal fundus findings who underwent MRI had normal imaging. ${ }^{6}$

In an Australian population, 693 patients with transient ischaemic attacks and acute stroke underwent pharmacological pupillary dilation and fundus photography for assessment of retinal microvascular signs (retinopathy, focal arteriolar narrowing, arteriovenous nicking and an enhanced arteriolar light reflex). All retinal microvascular signs were more prevalent in patients with transient ischaemic attacks or stroke than in control subjects, suggesting that fundus photography may provide useful information in the risk stratification of patients with suspected cerebrovascular disease. ${ }^{7}$

\section{WHAT ARE POTENTIAL APPLICATIONS FOR NON-MYDRIATIC FUNDUS PHOTOGRAPHY IN NEUROLOGY?}

It is often difficult to discern the details of the optic disc and other parts of the ocular fundus through undilated pupils. The main hurdle is the technical difficulty of using the ophthalmoscope. When this technical barrier is removed, neurologists, even without specific additional training, can use the ocular fundus examination more easily to make informed decisions regarding a patient's care.

Fundus photography in patients with uncontrolled hypertension, headache, vision complaints or suspicion of cerebrovascular disease may give important information that influences patient management. Subtle and sometimes even not-so-subtle fundus abnormalities 
may be missed with a cursory funduscopic examination in an outpatient clinic. For example, finding even mild optic disc oedema should prompt an urgent workup to exclude serious underlying causes of elevated intracranial pressure (eg, cerebral venous thrombosis, tumour, obstructive hydrocephalus) in a patient whose headache may have otherwise been misdiagnosed as primary. Finding optic disc pallor in a patient with unexplained neurological symptoms may suggest demyelinating disease or its mimics. It could also alert the clinician to obtain orbital MRI as well as brain imaging to exclude an orbital mass (eg, an optic nerve sheath meningioma). A patient who presents to clinic for stroke follow-up care with a blood pressure of 220/ $110 \mathrm{~mm} \mathrm{Hg}$ and is found to have optic disc oedema with retinal haemorrhages and exudates consistent with hypertensive retinopathy may prompt referral to the emergency department as a hypertensive emergency.

Fundus photography has also been used in patients with chronic neurological conditions, such as cerebrovascular disease and dementia. The brain and retina are similar in their embryological origin and physiological properties; this has inspired research into direct visualisation of small vessels of the retina as a surrogate for intracranial small vessels, which cannot be readily seen in vivo. The retinal vasculature can be seen non-invasively using fundus photography. Indeed, studies have shown a link between retinal vascular changes seen with fundus photography and stroke and dementia. ${ }^{8}$

\section{Medical education}

Academic medical centres are uniquely positioned to benefit from the acquisition of a non-mydriatic fundus camera, as the benefits may be more easily realised in a large provider group in collaboration with other clinicians and in the training of the next generation of medical professionals. Most medical students also report that they prefer to use fundus photographs over direct ophthalmoscopy, and $20 \%$ of students in one study reported that their primary reason for not performing an ocular fundus examination during a patient evaluation was discouragement by their preceptor. ${ }^{9} 10$ A photograph allows clinicians to evaluate the ocular fundus more easily, to emphasise the importance of the ocular fundus examination in the complete evaluation of many classes of neurological disease, and to point out key features of the ocular fundus to trainees in the context of an actual patient's evaluation. It is easy to share challenging cases or findings with other clinicians and to facilitate the diagnostic evaluation. ${ }^{11}$ Using fundus photography in medical education has the potential to restore enthusiasm for the ocular fundus examination in future generations of clinicians.

\section{Telemedicine}

The rise of telecommunications technology has introduced important telemedicine opportunities, including ocular fundus photography interpretation. ${ }^{12}$ The use of telemedicine in acute stroke has increased dramatically in recent years, despite significant regulatory and other barriers to its implementation. The success of 'telestroke' evaluations has built upon rapid access to a specialist in areas where the specialist is not readily physically available. Rapid fundus photograph interpretation through a teleophthalmology consultation may become standardised in the future. In the meantime, fundus photographs may be shared with other clinicians and colleagues electronically to help facilitate triage decisions.

\section{ABNORMALITIES OF THE OCULAR FUNDUS REQUIRING URGENT EVALUATION}

All patients presenting to a neurologist with a visual complaint should be seen by an eye care specialist as soon as possible. Furthermore, the neurologist is likely to encounter the following ocular fundus abnormalities relatively frequently, all of which generally indicate the need of an emergent or expedited workup.

\section{Optic disc oedema}

Optic disc oedema can be difficult to see with a direct ophthalmoscope in some patients; a fundus photograph may help. A fundus photograph may not clarify whether a patient has very subtle optic disc oedema, but can help to look for other funduscopic signs of optic disc oedema. These signs include blurring of the optic disc edges, filling in of the optic cup, peripapillary folds, obscuration of vessels as they cross the optic disc margin, peripapillary retinal haemorrhages, optic nerve head hyperaemia and venous congestion (figure 2). ${ }^{13}$ Photographs can also be very useful in distinguishing pseudopapilloedema from true optic disc oedema. For example, multifocal, irregular globules (sometimes described as tapioca-like) within the optic nerve head are diagnostic for optic nerve head drusen (figure 3).

\section{Optic disc pallor}

A normal optic disc is typically pink/orange in colour. An abnormally white optic disc appearance is referred to as optic disc pallor: a sign of damage to the optic nerve that takes at least 4-6 weeks to develop after an injury. The pallor may be subtle, and also assessment of optic disc colour is subjective and can be influenced by photographic exposure, adding to the difficulty in detecting optic disc pallor. In unilateral cases, it may be easier to detect pallor by comparing fundus photographs between the two eyes (figure 4). Details of the pallor may also help, such as definite pallor limited to the superior or inferior portion of the optic disc, as in patients with non-arteritic anterior ischaemic optic neuropathy. Optic disc pallor can provide additional evidence for optic neuritis in a patient with suspected demyelinating disease. Moderate-to-severe generalised pallor is accompanied by a noticeable lack of small 


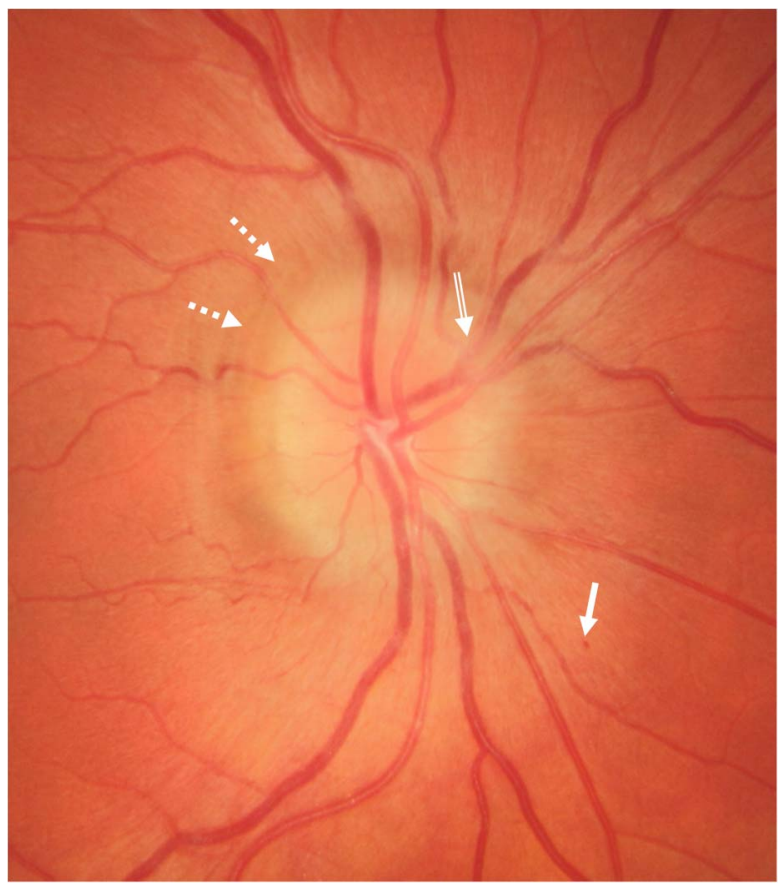

Figure 2 Papilloedema in a patient with idiopathic intracranial hypertension. This photograph shows several key findings that, in combination, are quite specific for true optic disc oedema, including peripapillary retinal folds (concentric arcs identified by dashed arrows), partial optic disc vessel obscuration (double arrow) and a small focal haemorrhage (arrow).

vessels on the temporal part of the optic disc. Thus, abnormal optic disc colour is not the only abnormality to suggest optic disc pallor.

\section{Central or branch retinal artery occlusion}

Patients with central retinal artery occlusion classically exhibit a macular cherry-red spot with an otherwise pale retina, as the clinician sees the intact choroidal blood supply though the central (thinnest) part of the macula (figure 5). There may also be interruptions of the blood column within the retinal arteries giving the appearance of train cars, so-called 'box-carring'. In 46 weeks, the retinal whitening resolves and the optic disc may appear pale with attenuation of the retinal arterioles. An acute branch retinal artery occlusion causes whitening of the portion of retina supplied only by the affected vessel. As in a central retinal artery occlusion, the whitening may only last several weeks and the only clue may be attenuated arterioles within the affected area. Counterintuitively, it may be difficult to detect widespread abnormalities such as the retinal whitening from a central retinal artery occlusion with a direct ophthalmoscope because the narrow field of view makes it difficult to compare affected and unaffected parts of the retina.

\section{Central or branch retinal vein occlusion}

Central retinal vein occlusion is typically associated with extensive flame-shaped haemorrhages throughout the ocular fundus, dilated and tortuous retinal veins and there may be optic disc oedema. Branch retinal vein occlusion gives the same signs restricted to the vascular drainage territory of the affected vein branch (figure 6, occlusion at double arrow). Branch occlusions typically occur at arteriovenous crossings, as in this case (box 1). Risk factors include increasing age, hypertension, diabetes mellitus, hyperlipidaemia, cigarette smoking and thrombophilia. ${ }^{14}$

\section{Severe hypertensive retinopathy}

It may be easier to see features of both mild and severe hypertensive retinopathy with fundus photography than with direct ophthalmoscopy. Hypertensive retinopathy is usually mild and is characterised by arteriolar narrowing as one of the earliest signs. Optic disc oedema occurs in very severe hypertensive retinopathy, and can usually be differentiated from other causes of optic disc oedema by the presence of retinal haemorrhages distant from the optic disc and exudates that indicate breakdown of the blood-retina barrier. Cotton wool spots (areas of retinal infarction) may also develop in severe cases.

\section{Intraretinal haemorrhages}

Intraretinal haemorrhages are a sign of blood vessel damage, most often from hypertension, but their significance is not completely clear. A trial of 497 patients presenting to a tertiary care academic emergency department with headache found that 42 had ocular fundus abnormalities, 15 of which were isolated retinal haemorrhages, presumably related to hypertension. Another 6 had grade III/IV hypertensive retinopathy, suggesting that half of the patients $(21 / 42)$ with abnormal ocular fundus findings in this headache population had findings related to hypertension.

\section{INTERPRETATION OF FUNDUS PHOTOGRAPHS BY NEUROLOGISTS}

Non-ophthalmologists are likely to interpret ocular fundus abnormalities more easily using photographs than with direct ophthalmoscopy. Nevertheless, instruction in the approach to the ocular fundus examination via photographs may help neurologists to use fundus photography in clinical care. While there is no one universally advocated approach, having a systematic and organised approach to the examination of the ocular fundus in a photograph has the potential to improve the accuracy of interpretation. Nevertheless, it cannot be overemphasised that an ophthalmologist must also evaluate all patients with vision loss, regardless of ocular fundus findings.

As with any other diagnostic test, it is best to interpret fundus photographs in the context of the clinical history and examination findings. Such context is often essential to know what findings may be incidental or inconsequential, and what others may 

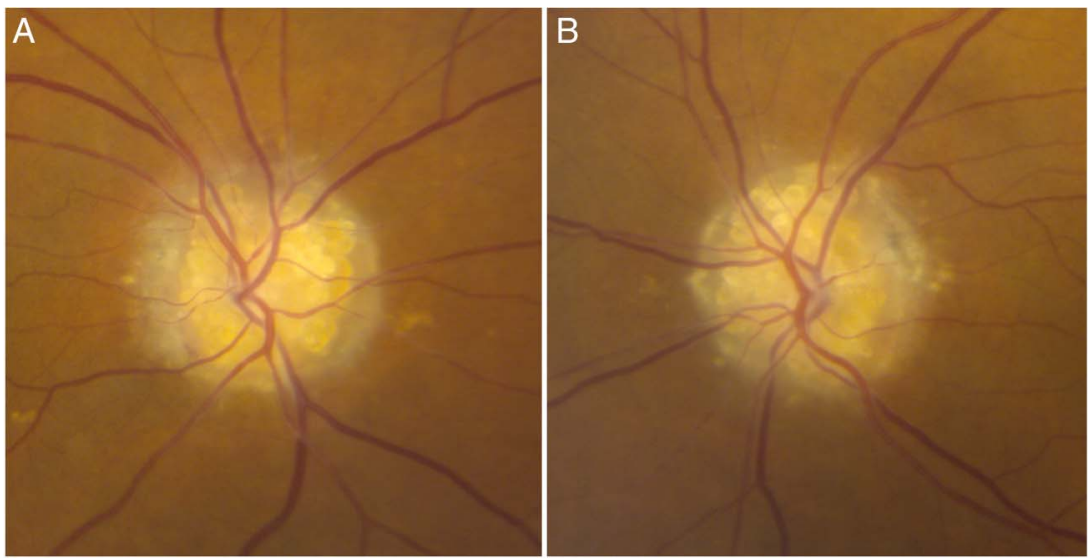

Figure 3 Prominent optic nerve head drusen. There are irregular, tapioca-like globules in this patient's optic nerve heads: right eye $(A)$ and left eye (B). Less obvious examples require careful examination of the optic nerve head to screen for this not uncommon mimic of optic disc oedema.

contribute valuable information to the patient's evaluation and inform further management decisions. ${ }^{15}$

As a generic approach to the ocular fundus examination in a photograph, one may arbitrarily divide the ocular fundus into regions: this prompts the clinician to look for specific findings in each region. Determining which eye you are viewing can be facilitated by remembering that the optic disc is closest to the nose. Therefore, in a properly centred photograph in which you see the macula and the optic disc, you will find the optic disc to the left side of the photograph in the left eye and to the right side in the right eye.

\section{Optic disc}

Key features of the optic disc to be evaluated during a fundus examination include the disc colour, sharpness of margins and the cup-to-disc ratio. In a photograph, the cup-to-disc ratio can be obvious, or difficult to discern, depending on the patient. An enlarged cup-to-disc ratio may be normal and physiological, or associated with glaucoma. The temporal portion of the optic disc is normally slightly more pale than the nasal portion, but excessive or generalised optic disc pallor can suggest optic neuropathy (figure 4). In the setting of a relative afferent pupillary defect, unilateral or asymmetrical optic disc pallor confirms damage to the optic nerve that began at least 4-6 weeks before. Blurring of the optic disc margins is a sign of optic disc oedema, and the differential diagnosis is influenced by whether the oedema is segmental or generalised, and unilateral or bilateral.

\section{Macula}

The macula is normally found temporal and slightly inferior to the optic disc and contains densely packed cone photoreceptors that are essential for high-acuity colour central vision. The foveal light reflex is a normal reflection of light from the thin retinal layers at the fovea and can sometimes be seen in fundus photographs. A decreased foveal light reflex in one eye associated with a decrease in visual acuity in that
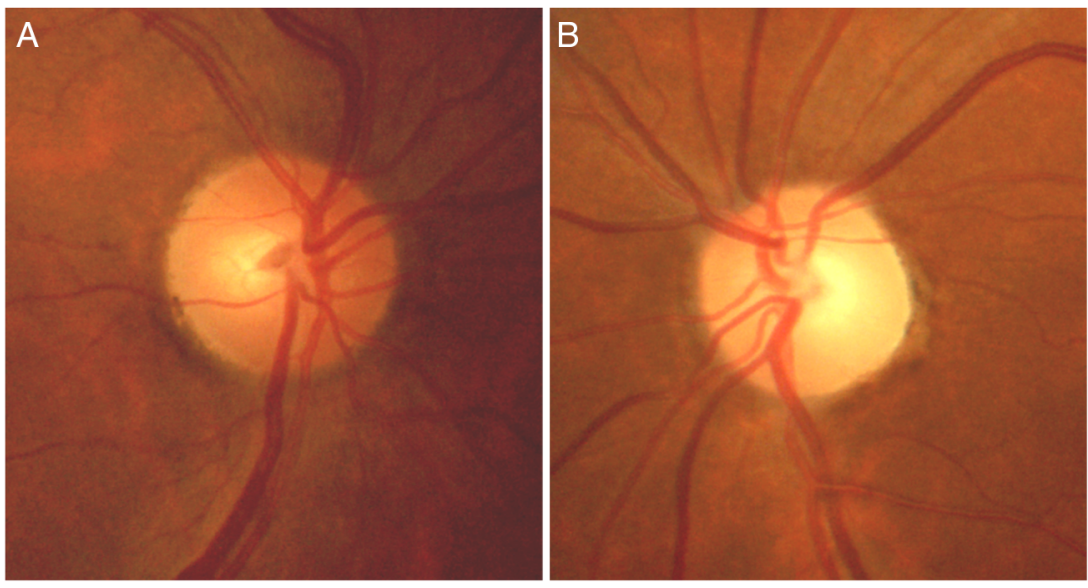

Figure 4 Unilateral optic disc pallor. The right optic disc (A) is normal in colour. The left optic disc (B) exhibits subtle pallor, particularly temporally (right side of the disc in the photograph). Comparing the optic discs from each eye in photographs is a valuable tool in the detection of unilateral optic disc pallor. 


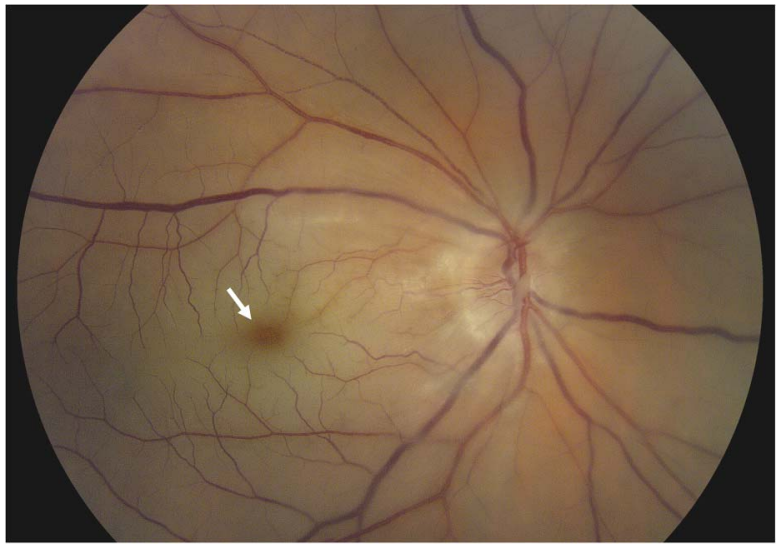

Figure 5 Central retinal artery occlusion. Note the macular cherry red spot (arrow) with an otherwise pale retina, as the clinician can see the intact choroidal blood supply though the central (thinnest) part of the macula.

eye can indicate a foveal abnormality, such as macular oedema. Neuroretinitis, caused by inflammation of the retina and optic disc, is primarily recognised by an associated macular-star pattern of exudates, often with optic disc oedema (figure 7). Other macular abnormalities such as haemorrhage are generally more easily seen with fundus photographs than via direct ophthalmoscopy.

\section{Retinal vessels}

Dilated retinal vessels may develop in papilloedema or in occlusive disorders of the retinal veins, such as a central or branch retinal vein occlusion. Arteriovenous nicking may occur in patients with chronic hypertension, in which a hard atherosclerotic arteriole

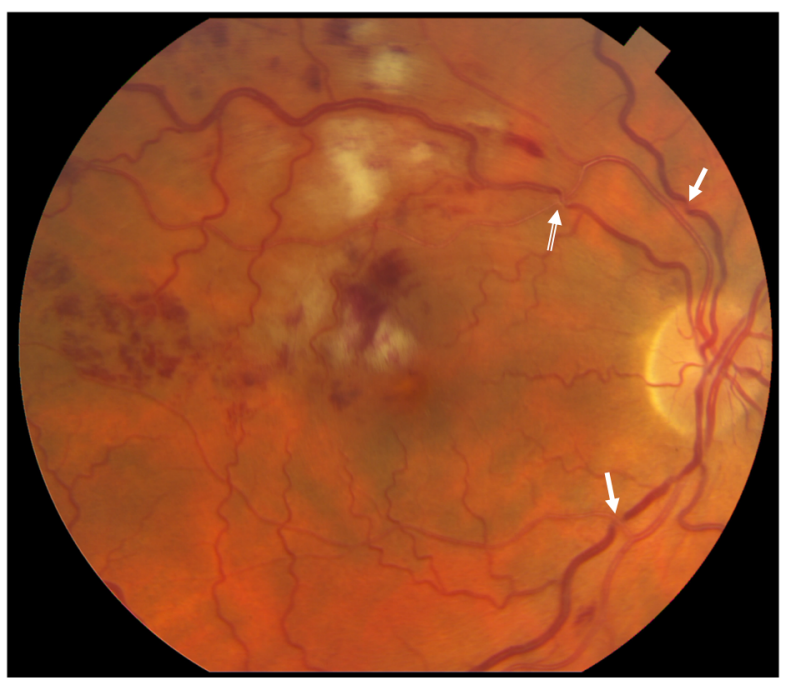

Figure 6 Branch retinal vein occlusion (BRVO). Note the presence of haemorrhages, cotton wool spots and tortuous and dilated veins distal to the site of BRVO (double arrow) involving the superotemporal macula. Arteriovenous nicking is also seen as a result of uncontrolled systemic hypertension (arrows).

\section{Box 1 Case study}

A 40-year-old woman presented for evaluation of probable idiopathic intracranial hypertension (IIH). She has a history of refractory hypertension and headaches. She had several months of episodic vision disturbances with lightheadedness and worsening of headaches. An MRI of the brain showed an empty sella turcica. A neurologist diagnosed $\mathrm{IH}$, without a lumbar puncture at that time. Several months later, she noticed a dark spot in the vision of her right eye. Her neurologist then arranged for a lumbar puncture, which showed an opening pressure of $22 \mathrm{~cm}$ of water with normal cerebrospinal fluid. The neurologist documented 'mild blurring of the disk margins' and started her on acetazolamide for a presumed vision disturbance related to IIH.

On examination, her body mass index was $43.1 \mathrm{~kg} / \mathrm{m}^{2}$ and blood pressure was initially $216 / 143 \mathrm{~mm} \mathrm{Hg}$. Visual acuity was $6 / 60$ in the right eye and $6 / 6$ in the left eye. There was no relative afferent pupillary defect. Confrontation visual fields showed decreased vision inferonasally in the right eye and were full in the left eye.

Fundus photographs showed pink, sharp optic discs with no disc oedema, but the right eye retina showed extensive retinal haemorrhages localised to the superotemporal macula with scattered flame-shaped haemorrhages and cotton wool spots in the same localised area, in a pattern consistent with a branch retinal vein occlusion (figure 6).

$\mathrm{IIH}$ had been misdiagnosed in this case, partially because of difficulty performing funduscopy. It was presumed that her vision disturbance was from IIH, although a fundus photograph clearly showed significant retinal abnormalities without optic disc oedema that would have been easily discerned by a non-ophthalmologist assisted by a photograph. A fundus photograph performed by the referring neurologist would have localised the abnormality, which was not consistent with IIH, prevented unnecessary invasive testing, and facilitated referral to an ophthalmologist for evaluation and management.

compresses a retinal vein, resulting in a focal change in the contour of the vein and a 'nicked' appearance (figure 6, arrows). Patients with a history of transient monocular vision disturbance should be examined for a Hollenhorst plaque, which is a sign of cholesterol embolisation from a proximal atherosclerotic source (figure 8).

\section{Mid-peripheral retina}

Retinal abnormalities such as haemorrhages, exudates and microaneurysms are often difficult to detect with a direct ophthalmoscope and may be more easily detected in a fundus photograph. Haemorrhages and exudates distant from the optic disc can occur in 


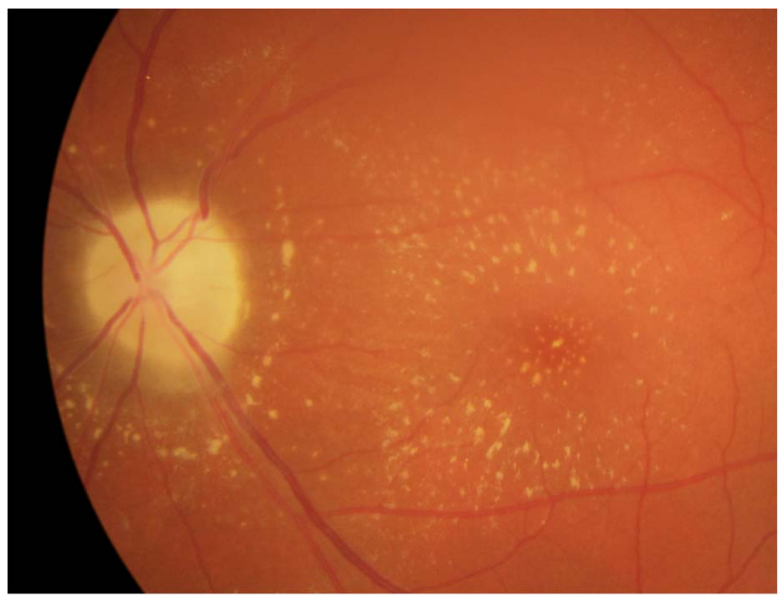

Figure 7 Neuroretinitis. This photograph depicts optic disc pallor for optic nerve damage as well as yellowish exudates in the peripapillary retina and macula that are radially oriented in a 'star' pattern, consistent with neuroretinitis. Optic disc oedema may be the first sign, followed days to weeks later by retinal exudates suggestive of retinal inflammation.

conditions such as hypertensive retinopathy and diabetic retinopathy and would not be expected in other conditions, such as papilloedema from raised intracranial pressure. Microaneurysms appear as small dots of blood in the retina due to outpouching of retinal capillaries, and can occur in diabetic retinopathy.

\section{Other normal findings}

A highly reflective layer, sometimes surrounding the optic nerve and macula, may be visible in photographs in younger patients. This is the inner limiting

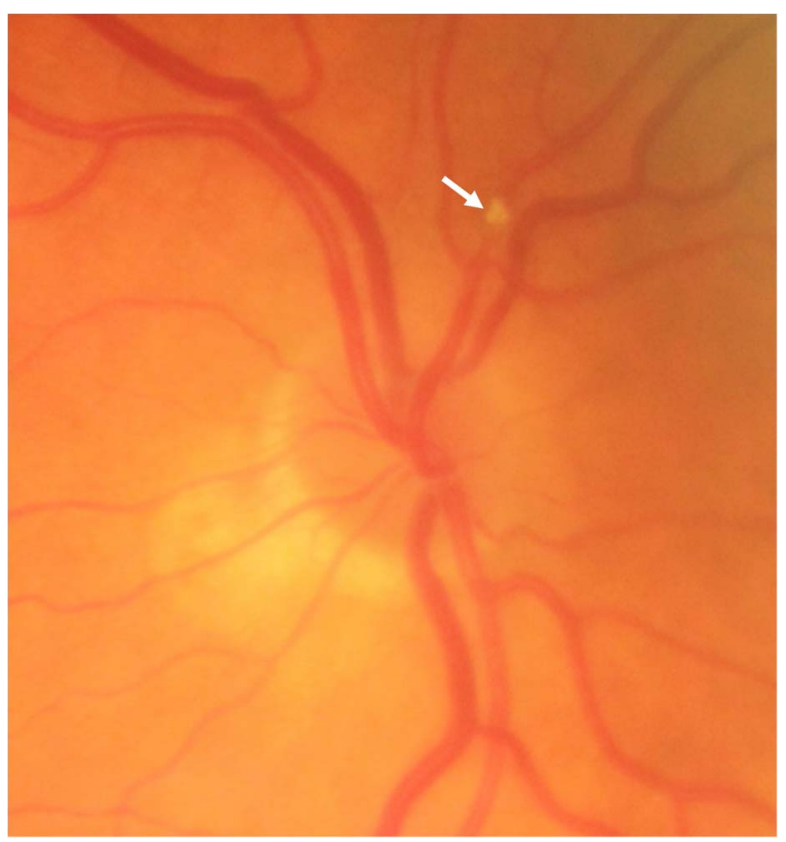

Figure 8 Hollenhorst plaque. There is a cholesterol embolism (Hollenhorst plaque, arrow) in a superior retinal arteriole, which came from a proximal atherosclerotic source. membrane, and is a normal finding that may confuse some non-ophthalmologists. Patients with lightly pigmented skin can have a distinctive ocular fundus appearance with 'tangles' of orange streaks behind the retinal vessels, which are the choroidal vessels, and are a normal finding called a 'blonde' fundus. Conversely, patients with more darkly pigmented skin tend to have a more 'tigroid' appearance that resembles tiger stripes, which represents the choroidal vessels against a background of darker pigments, which is also a normal finding.

\section{Artefacts}

Artefacts are common in fundus photography and may result from incorrect patient-camera orientation, excessively small pupils, camera lens debris or other causes. A faded whitish area with a gradient from the edge of the photograph may be a sign of artefact from the pupil border in patients with smaller pupils. There may be other cloudy artefacts, confirmed as artefactual by comparing with photographs from the same eye in a slightly different position (in which the artefact appears in a slightly different location), or from the contralateral eye.

\section{WHAT ARE ADVANTAGES AND DISADVANTAGES OF NON-MYDRIATIC FUNDUS PHOTOGRAPHY IN NEUROLOGY? \\ Strengths}

As with any diagnostic tool, an accurate understanding of the strengths and limitations of non-mydriatic fundus photography can help clinicians to know when fundus photographs may be useful. A major advantage is convenience. Obtaining images from a fundus camera in the emergency department or an outpatient clinic is relatively fast and easy, with the median time of image acquisition in phase I of the FOTO-ED study being just $1.9 \mathrm{~min}^{4}$ As handheld non-mydriatic fundus camera technology improves and becomes more commonplace, it should help one to obtain a view of the ocular fundus while avoiding pharmacological pupil dilatation in critical care patients with intracranial pathology in whom following a pupillary examination can be clinically valuable. Clinicians and students also find fundus photograph interpretation easier than using a direct ophthalmoscope through an undilated pupil. ${ }^{5} 910$ The quality of the images is also very good in most patients, rivalling the quality of mydriatic fundus cameras. The resolution of the images typically also allows for digital zoom to appreciate finer details of the ocular fundus. As was also shown in phase I of the FOTO-ED study, nurse practitioners who operated the fundus camera required minimal training. The images are also available to share with others for educational purposes, to facilitate triage decisions and to enhance medical education. ${ }^{11}{ }^{16}$ The photographs may also serve as a form of documentation, 
which may help in future clinic visits and medicolegal cases. For the purposes of a neurology evaluation, non-mydriatic fundus photography allows for a detailed assessment of the ocular fundus, without pupillary dilatation.

\section{Limitations}

One disadvantage or limitation of non-mydriatic fundus photography is the cost of equipment, which can be around $£ 17000-£ 22000$ (\$25 000-\$30 000) depending on the model. However, prices are likely to fall with more widespread adoption of the technology. Also, current non-mydriatic cameras require a patient to be alert and cooperative, to sit still and to be able to follow simple commands, which applies to most outpatients, but not all. There are several models of handheld non-mydriatic cameras that may be useful for uncooperative patients in acute care settings, such as the intensive care unit, although the quality of images is currently inferior to stationary desktop models and the devices are considerably more difficult to use. Devices that attach to smartphones to allow fundus photographs to be taken have become more popular in recent years, but they generally still require pupillary dilatation for adequate views of the ocular fundus, which limits their use among neurologists. Non-mydriatic fundus photographs also do not provide a dynamic view of the ocular fundus, meaning that the evaluation of spontaneous venous pulsations, subtle abnormalities of visual fixation such as low amplitude nystagmus, or the rare occurrence of tracking a moving retinal embolus remain reserved for a live funduscopic view via an ophthalmoscope or the occasional camera with video capabilities.

\section{CONCLUSION}

The ocular fundus examination is still important, despite the demise of direct ophthalmoscopy in recent

\section{Key points}

- Non-mydriatic fundus photography is an effective alternative to direct ophthalmoscopy for examining the ocular fundus in non-ophthalmology settings.

- Even without additional training, it is easier to detect and interpret abnormalities of the ocular fundus with photographs rather than with direct ophthalmoscopy.

- With increasing availability of non-mydriatic fundus photography, neurologists need to become familiar with its advantages and limitations in clinical care.

- Rapidly advancing technology is improving both the quality and availability of non-mydriatic fundus photography.

- Fundus photography has the potential to improve medical education about the ocular fundus examination. decades. The non-mydriatic fundus camera is an alternative tool that bypasses the technical difficulty of the direct ophthalmoscope and makes the ocular fundus examination more accessible. A combination of clinician discomfort with the use of a direct ophthalmoscope, persistent relevance of the ocular fundus examination and increasing availability of nonmydriatic fundus cameras in clinical care is creating momentum for more widespread adoption of this technology. Neurologists are poised to benefit from this momentum and should be familiar with the application of non-mydriatic fundus photography in clinical care.

Contributors All authors contributed to the writing and final approval of this manuscript.

Funding Research to Prevent Blindness, National Institute of Neurological Disorders and Stroke (R01-NS089694), National Eye Institute (P30-EY006360).

Competing interests None declared.

Provenance and peer review Commissioned; externally peer reviewed. This paper was reviewed by Christian Lueck, Canberra, Australia.

\section{REFERENCES}

1 Mackay DD, Garza PS, Bruce BB, et al. The demise of direct ophthalmoscopy: a modern clinical challenge. Neurol Clin Pract 2015;5:150-7.

2 Lamirel C, Bruce BB, Wright DW, et al. Quality of nonmydriatic digital fundus photography obtained by nurse practitioners in the emergency department: the FOTO-ED study. Ophthalmology 2012;119:617-24.

3 Bruce BB, Lamirel C, Wright DW, et al. Nonmydriatic ocular fundus photography in the emergency department. N Engl J Med 2011;364:387-9.

4 Bruce BB, Lamirel C, Biousse V, et al. Feasibility of nonmydriatic ocular fundus photography in the emergency department: phase I of the FOTO-ED study. Acad Emerg Med 2011;18:928-33.

5 Bruce BB, Thulasi P, Fraser CL, et al. Diagnostic accuracy and use of nonmydriatic ocular fundus photography by emergency physicians: phase II of the FOTO-ED study. Ann Emerg Med 2013;62:28-33.

6 Thulasi P, Fraser CL, Biousse V, et al. Nonmydriatic ocular fundus photography among headache patients in an emergency department. Neurology 2013;80:432-7.

7 Wang JJ, Baker ML, Hand PJ, et al. Transient ischemic attack and acute ischemic stroke: associations with retinal microvascular signs. Stroke 2011;42:404-8.

8 Cheung CY, Chen C, Wong TY. Ocular fundus photography as a tool to study stroke and dementia. Semin Neurol 2015;35:481-90.

9 Kelly LP, Garza PS, Bruce BB, et al. Teaching Ophthalmoscopy to Medical Students (the TOTeMS Study). Am J Ophthalmol 2013;156:1056-61.

10 Mackay DD, Garza PS, Bruce BB, et al. Teaching Ophthalmoscopy to Medical Students (TOTeMS) II: A One-Year Retention Study. Am J Ophthalmol 2014;157:747-8.

11 Mackay DD, Garza PS. Ocular fundus photography as an educational tool. Semin Neurol 2015;35:496-505.

12 Pérez MA, Bruce BB, Newman NJ, et al. The use of retinal photography in nonophthalmic settings and its potential for neurology. Neurologist 2012;18:350-5. 
13 Carta A, Favilla S, Prato M, et al. Accuracy of funduscopy to identify true edema versus pseudoedema of the optic disc. Invest Ophthalmol Vis Sci 2012;53:1-6.

14 Rehak J, Rehak M. Branch retinal vein occlusion: pathogenesis, visual prognosis, and treatment modalities. Curr Eye Res 2008;33:111-31.
15 Woodward MA, Bavinger JC, Amin S, et al. Telemedicine for ophthalmic consultation services: use of a portable device and layering information for graders. J Telemed Telecare. Published Online First: 1 March 2016.

16 Bidot S, Bruce BB, Newman NJ, et al. Nonmydriatic retinal photography in the evaluation of acute neurologic conditions. Neurol Clin Pract 2013;3:527-31. 